\title{
Renal Vasculature in Essential Hypertension: A Review of Some Contrarian Evidence
}

\author{
Richard E. Tracy
}

Department of Pathology, Louisiana State University Health Sciences Center, New Orleans, La., USA

\begin{abstract}
This chapter reviews a body of evidence concerning the anatomic pathology, pathogenesis, epidemiology and possible etiologic agents of benign essential hypertension in human populations. A core finding serves as the starting point for further reasoning: intimal fibroplasia of renal interlobular arteries (arteriosclerosis) increases with age at varying rates in all populations around the world, and the rise of mean arterial pressure (MAP) with age is closely tied to this process. The weight of evidence favors the view that fibroplasia progresses for wholly unknown reasons, is not accelerated by elevations in MAP, and that it raises MAP in proportion to its severity by creating nephron heterogeneity that initiates Goldblatt mechanisms. This form of hypertension has been designated type 2 to distinguish it from a less common form, called type 1, where the fibroplasia is of mild or minimal degree. This chapter reviews evidence that indicates type 1 does not evolve into type 2 because hypertension is not accelerating the process. Experimental models of hypertension include the Goldblatt model, which resembles some aspects of type 2, and spontaneous genetic models, which mimic some aspects of type 1 hypertension. There is currently no persuasive evidence that type 2 hypertension, as it naturally develops in the human during aging of 50 years or more, can be reproduced in laboratory animals. Clues to the etiology of the arterial fibroplasia that appears to underlie most instances of essential hypertension would best be sought in the study of variations among human populations around the world, and especially of their migrants.
\end{abstract}

Copyright ๑ 2011 S. Karger AG, Basel
The features of the renal arterial tree in human hypertension have gathered no consensus descriptions of their anatomy or of their relationships to blood pressure. The review here considers some of the contrarian evidence that emphasizes perspectives outside of most mainstream viewpoints.

The disease entity, hypertension, is named for a clinical sign, not for an etiologic agent or pathognomonic lesion, or even a symptom. This odd circumstance is at least in part due to the lack of clear understanding of the anatomic or biochemical abnormalities that may properly belong to this entity.

Much is said about medial hypertrophy in the arterial tree. This usage is sometimes correct, but is seriously misdirected in other circumstances. Patients with long-standing hypertension followed at autopsy show a pattern of arterial features summarized in figure 1a [1]. Medial hypertrophy, attributable chiefly to smooth muscle cell (SMC) enlargement, typifies the arteries that serve mainly as a conduit function (i.e. generally those with diameters greater than $0.3-0.5 \mathrm{~mm}$ ). Resistance level arteries, however, with diameters less than $0.3 \mathrm{~mm}$, display medial hypertrophy rarely and only in special circumstances. These typically undergo remodeling to 
a smaller caliber while preserving an unchanged medial muscle mass, thereby increasing the wall thickness to diameter ratio (eutrophic inward remodeling [2]).

This pattern affects the arteries within the bulk tissues of the body, skin, muscle, fat, bone, etc., and therefore accounts for the increased peripheral resistance that persists in the presence of normal or slightly increased cardiac output. This increased resistance serves to raise the sheer stress needed to disperse the energy of high pressure as heat so that locally autoregulated capillary flow is maintained at normal pressures. Some data have suggested that the afferent arterioles of hypertensive kidneys also participate in this remodeling to smaller calibers in humans [3] and, from indirect evidence, in rats with spontaneous hypertension $[2,4]$.

Arteries within the renal cortex are essentially all in the diameter range less than $0.3 \mathrm{~mm}$, and therefore do not manifest medial hypertrophy. Medial SMC hypertrophy can sometimes be seen in glomerulonephritis, but in no other circumstances [5]. This observation supports that the hypertrophy can be used as a helpful diagnostic criterion for recognizing glomerular disorders. This principle might explain the medial hypertrophy that often accompanies glomerulosclerosis in spontaneously hypertensive rats [6]. In benign essential hypertension, alterations of arteries and arterioles are mainly of the kinds illustrated in figures $1 \mathrm{~b}-\mathrm{e}$.

Of major concern to the topic of benign essential hypertension is the structural alteration illustrated in figure $1 \mathrm{~b}$. This has been called arteriosclerosis, arteriolosclerosis, arterial intimal fibroplasia (fibroplasia for short), and other names. Fishberg [7] noted in 1925, 'As far back as 1868 , Johnson described hypertrophy of the media as a characteristic in granular kidney and most current textbooks contain the same statement, but, as a matter of fact, one finds in arteriosclerosis a gradual and progressive degenerative atrophy of the medial muscle with replacement fibrosis'. More than 80 years later, this statement is still valid today. This arteriosclerosis is rarely seen in more than a minimal degree in the bulk tissues of the body; it is most severe and extensive in the kidney, although it is often found in the pancreas, spleen, liver and other abdominal organs as well, but generally not the gastrointestinal tract. Related kinds of change sometimes affect small arteries of the brain and heart.

The condition shown in figure $1 \mathrm{~b}$ is a kind of metaplasia, wherein medial tissue withers and disappears, and is replaced by a newly formed intima molded of cell-poor, densely compacted collagen and associated matrix. It should be noted that in comparison with a normal artery, the span from positions 1 to 2 in the two examples display equally about 8 or 9 SMC nuclei, as if the intimal cells in the fibroplasia are the pre-existing medial SMCs that have altered size, orientation, shape and inter-relationships. In figure $1 \mathrm{c}$, the features suggest that a collagenous zone has formed within the mid-depth of media displacing some of it inward $(\mathrm{z})$, retaining the original internal elastica $(\mathrm{x})$ and forming a new one for the remnant outer media (y). If this is the usual pattern of progression, then the inner remnant of media nearly always soon vanishes, perhaps by transformation into additional intima. In early stages, the fibrotic media can have increased thickness [8], which can deceptively be mistaken for hypertrophy (or even for hyperplasia). There is clearly no indication of hyperplasia, although a quantitative test of this question has never been reported. For purposes of measurement, the best results are obtained from measuring intimal thickness rather than wall thickness because the thickness of a shrinking media countervails the process of interest and dilutes useful information. Intima as a percentage of outer diameter is called $\mathrm{R}, \mathrm{R}_{\mathrm{C}}$ applying to diameters of $150-300 \mu \mathrm{m}$ and $R_{R}$ to those of $80-149 \mu \mathrm{m}$.

Something very different occurs in malignant hypertension. A major diagnostic finding is the mucinous edema of an expanding intima affected by SMC proliferation, lymphocytic and PMN infiltration, and other features (fig. 1d) [9-11]. It is easy to confuse this change with the benign condition, which might explain the faulty use of the term hyperplasia to describe the benign 

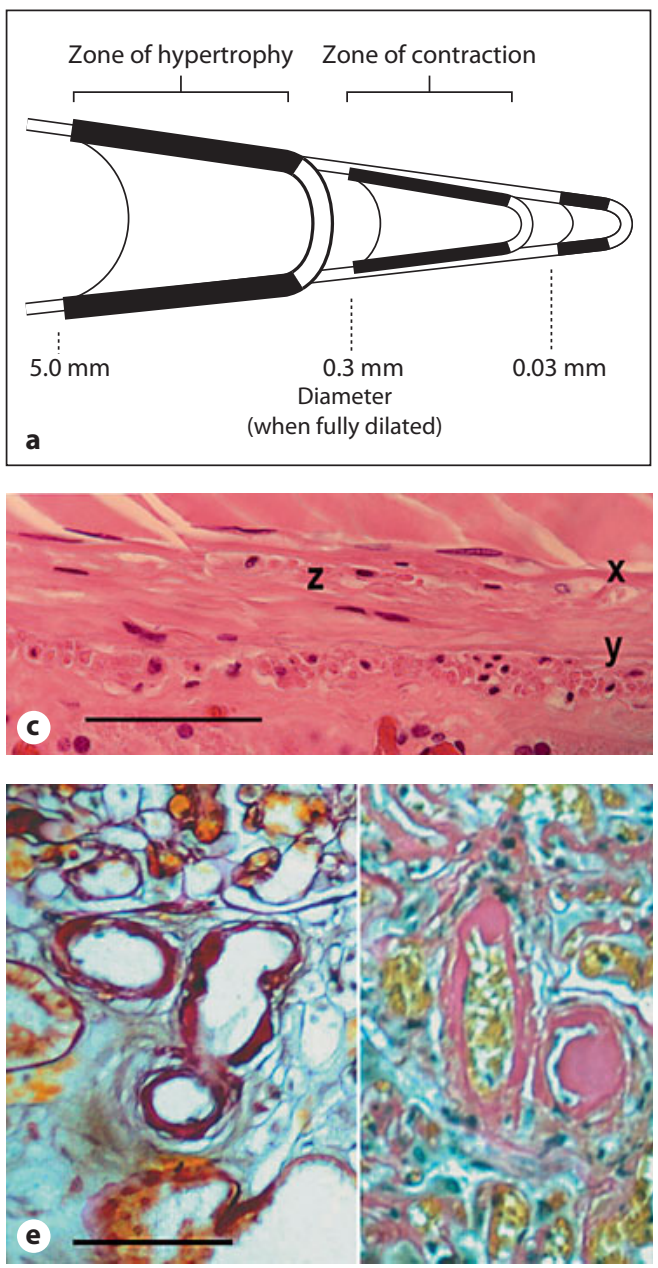
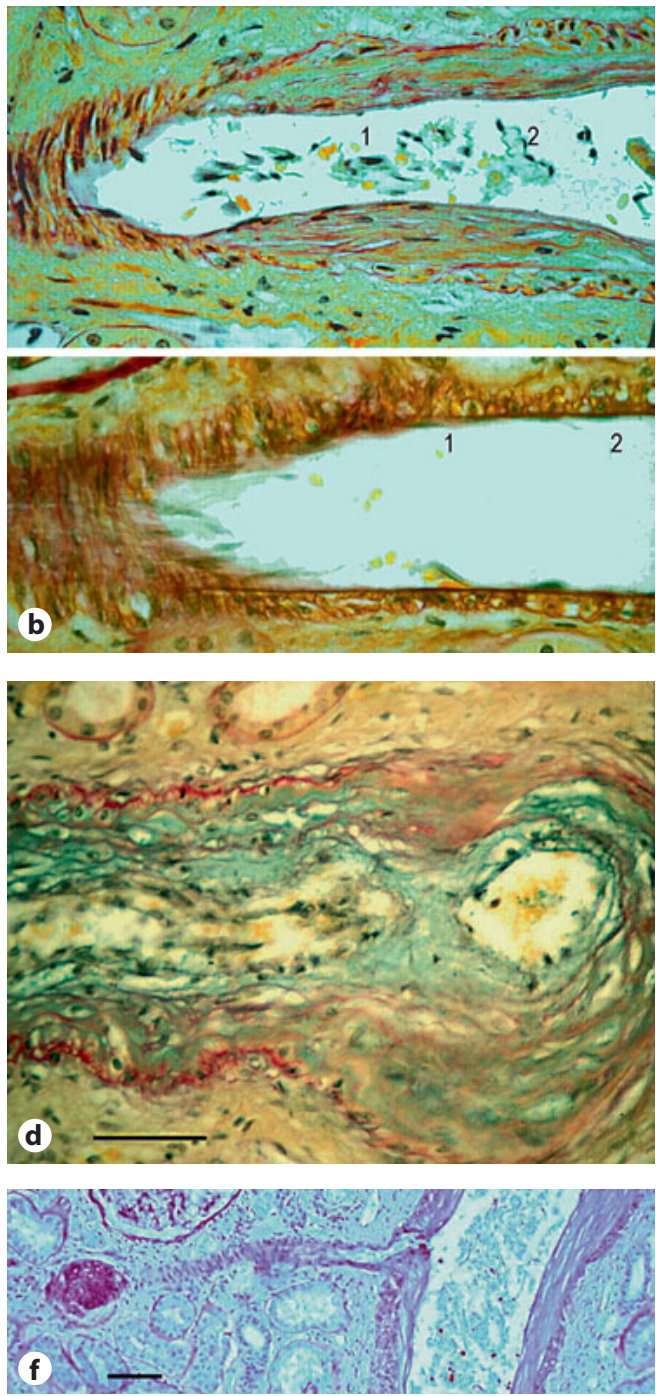

Fig. 1. a Arterial features of patients with long-standing hypertension [1]. b Fibroplastic (upper) and normal (lower) interlobular arteries. c Interlobular artery with benign features. d Interlobular artery with malignant features. e Pressure-fixed and immersion-fixed afferent arterioles with hyalinization; glomeruli at top of fields. $\mathbf{f}$ Obsolete glomerulus with arteriolar constriction at the ostium of its afferent arteriole. Frames b, d, e and f: PAS-Alcian blue. Frame c: H\&E. Bars $=60 \mu \mathrm{m}$.

condition. Autoregulation of renal blood flow can fail when MAP exceeds $160 \mathrm{~mm} \mathrm{Hg}$, severely increasing flow rates, and the consequent excessive sheer stress may be the cause of these malignant structural changes.

The hyalinization of afferent and efferent arterioles $[12,13]$ and of terminal arteries (diameter $<80 \mu \mathrm{m}$ ) is almost universally described as constricting or occluding the lumen, causing nephron atrophy and nephrosclerosis. However, when formalin is perfused under pressure into the renal artery, these deposits are pressed outward to restore a fully intact lumen (fig. 1e) [3]. When lacking pressure, the hyaline deposits 
Fig. 2. Each dot represents a case and the Maltese crosses are the twodimensional means. Reproduced from [16].

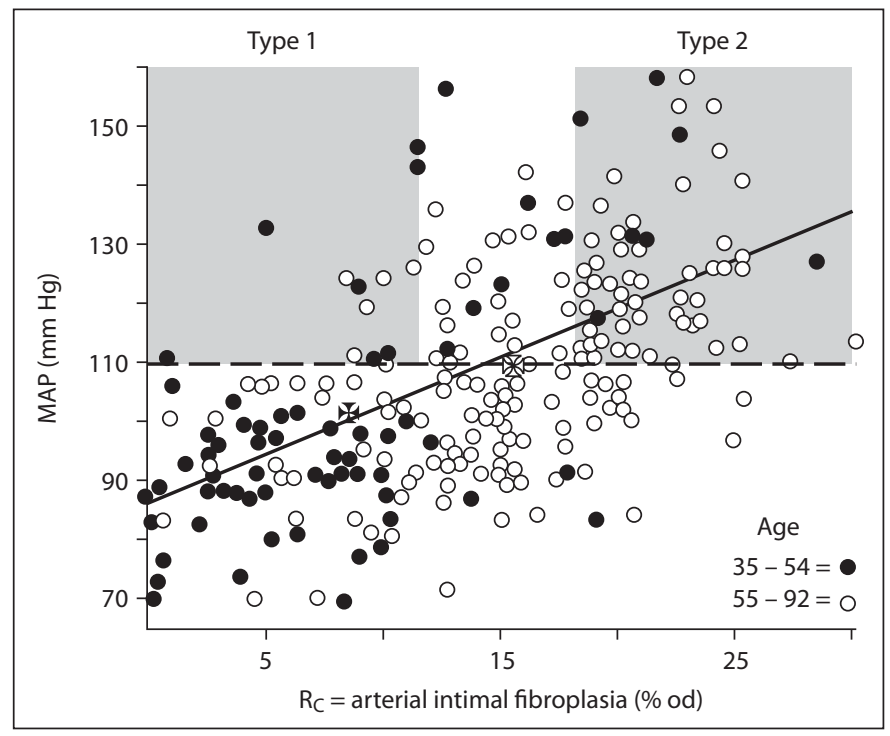

take a rounded form like that of liquid droplets. When pressure is restored, they reform back into flattened components of the vessel wall with outward bulging, reminiscent of the outward remodeling at sites of atherosclerotic plaques. Rather than hyalin as a cause of nephron atrophy, it may be that the arteriole is constricted as it originates through the fibroplastic intima of its parent artery, a suggestion that has some observational support [14] (fig. 1f). Hence, it may be that the fibroplasia fosters fibrous replacement of atrophied nephrons (nephrosclerosis) [15] because of these strictures at ostia of afferent arterioles. Features of atherosclerosis can occur in arteries as small as $500 \mu \mathrm{m}$ (arcuates), but never at sizes $<300 \mu \mathrm{m}$ (interlobulars). Atherosclerotic materials in arteries of these sizes are attributed to emboli from a diseased aorta.

Some years ago, a series of autopsies was assembled by reviewing outpatient clinical records of patients at autopsy [16]. Those records sometimes provided at least 3 years of outpatient blood pressures [MAP (mean arterial pressure)= $(2$ diastolic + systolic $) / 3$ ] and at least 10 readings, and these cases were retained for measurement of fibroplasia in interlobular arteries. A transition from low to high or from high to low MAP (at least to some minimal degree, but often of a large amount), was identified in all records. It was subsequently found that only the levels after transition were of interest, and the earlier records offered no useful additional information. Figure 2 summarizes the pertinent findings for the present purposes.

MAP correlates with $R_{C}$ in accordance with a linear regression that applies to men and women of all ages in the range 35-74 years ( $\mathrm{r}$ $=0.6$ ). Taking an arbitrary MAP cutoff of 110 $\mathrm{mm} \mathrm{Hg}$ for hypertension, it is possible to recognize two types of hypertension. Type 2 occurs in association with severe degrees of fibroplasia, while type 1 reveals only mild or minimal degrees. This range of variation has been recognized since the early 20th century [7]. An especially influential demonstration of this spectrum was provided by Sommers et al. [17]. While performing lumbar sympathectomy in hopes of reversing hypertension, they obtained tiny superficial kidney biopsies to evaluate for arterial and arteriolar abnormalities. The authors were especially impressed by the finding of some hypertensives with mild or minimal abnormalities. Although they did not use the terms type 1 and type 2 , these terms could 
Fig. 3. Each dot represents a case. Reproduced from [16].

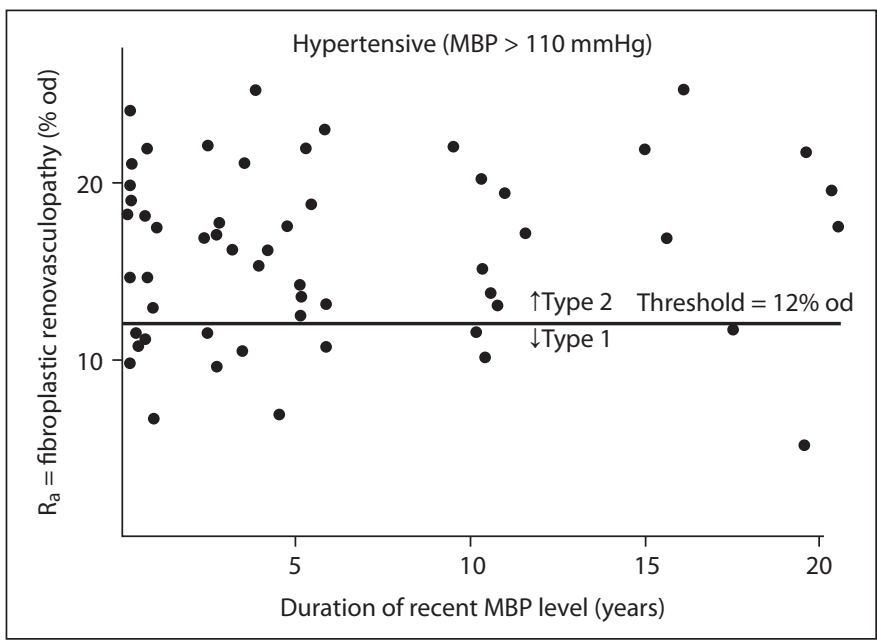

have been introduced from their findings. In their conclusions we read, 'Although two thirds of the cases revealed advanced renal arteriolar sclerosis, the presence of even a few individuals with apparently normal renal vessels is consistent with the view that hypertension precedes structural changes in the kidney vasculature.' However, what was left unsaid is that these findings are also consistent with the possibility that the type 1 hypertensives will remain type 1 and experience no more than average rates of fibroplasia progression, or perhaps less, as they age.

Whether type 1 hypertension will fail to convert into type 2 over time (by analogy with types 1 and 2 diabetes) could best be determined with a prospective epidemiological study. This is not practical, however, because a biopsy at the beginning of such a study would be unethical. Biopsies available to Sommers et al. [17] were unique to those circumstances and have not been reproduced; those studies did not include later follow-up with autopsies. Figure 3, however, offers an approximation to the optimal study. Subjects whose lengthy records of MAP documented the onset of hypertension provided subsequent MAP readings with sustained hypertension for intervals ranging from 1-19 years [16]. At autopsy, the fibroplasia did not correlate with the duration of the persistently elevated MAP. Nine of these subjects with observed onset of hypertension were classified as type 1 after endurance of the hypertension for up to 19 years. This outcome offers the possibility that some type 1 subjects will remain type 1 , implying that hypertension does not accelerate fibroplasia. The findings in figure 3 provide evidence for the feasibility of such a study.

In recent times it has become possible to measure renal blood flow using Doppler ultrasound methods [18]. These methods have consistently identified what might be called type 1 and type 2 hypertensives on the basis of normal or diminished flow rates. The question of the progression of fibroplasia in type 1 hypertensives is therefore accessible to an answer with the use of autopsy follow-up. This would not be an ideal study because many of the hypertensives with normal renal blood flow are likely to have nephron heterogeneity based on fibroplasia unevenly distributed among the nephrons [19-21]. The outcome of this proposed study would therefore rely on whether or not a subset of hypertensives with persistent type 1 hypertension would be found at autopsy, after experiencing many years of sustained hypertension, thus reproducing the provisional conclusion of figure 3 .

Sealy et al. [21] have explained how such heterogeneity is uniquely structured to induce both high and low renin hypertension even in the 
Fig. 4. A simplified summation of the proposed thesis. Arteriosclerosis is unevenly scattered and generates one subset of nephrons that are ischemic and releasing renin, and another subset that are hyperemic and retaining sodium in response to the renin. The mean rate of function of all nephrons averaged can be within the normal range and clearance studies can fail to detect that kidneys are abnormal.

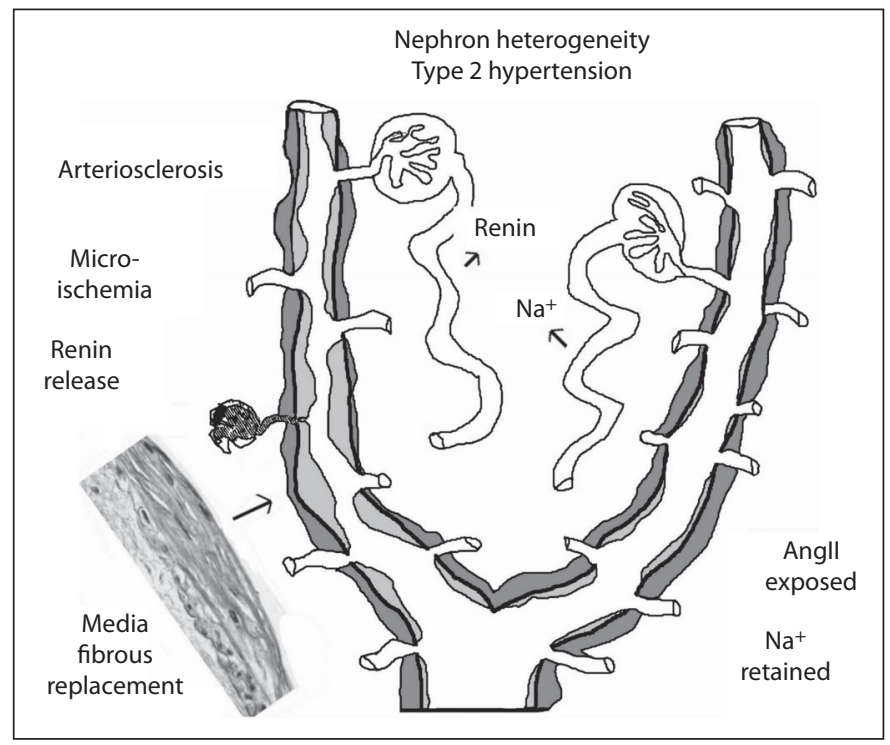

presence of normal overall flow rates. Figure 4 outlines the main features of this theory whereby ischemic nephrons supplied by sclerotic arteries release renin which then acts on the hyperemic nephrons supplied by competent arteries to cause sodium retention. Renin will be high if sodium is deficient and low if sodium space expansion is sustaining the hypertension.

Washout curves for radioactive xenon [19, 20, 22] have been used to partition renal blood flow into a fast and a slow component. It is customary to call these components cortical and juxtaglomerular, respectively, but Ladefoged [20] has noted, 'It is possible that areas in the cortex with low perfusion may be represented in the second component of the wash-out curve. . . . Data consistent with this possibility have been repeatedly reported by multiple observers $[19,22]$ : the slow component acquires an increasingly greater share of renal flow, and may sometimes show increases in its total amount, while the fast component declines dramatically with progressive disease. This outcome affirms an important prediction of the nephron heterogeneity hypothesis. This washout method has been challenged as lacking credibility [23].
After assembling the autopsy series in figure 2, the series was expanded by adding subjects from New Orleans, Bogalusa, Greenland and Bombay (Mumbai), India. Two regression equations, one relating MAP to $\mathrm{R}_{\mathrm{C}}$ and the other to $R_{R}$, were suitable to all populations equally well [24]. Using those equations, MAP was estimated from renal fibroplasia measured in autopsy specimens from New Orleans, Bombay and Tokyo [16] (fig. 5). Figure 5 also displays the trends of MAP revealed in national survey data. The patterns of MAP rising with age are nearly identical to those of renal arterial fibroplasia in these populations.

The patterns in figure 5 might seem equally consistent with the proposals that fibroplasia is the cause or the consequence of sustained hypertension. It should be noted, however, that fibroplasia begins at least as early as 20 years of age, at a time when hypertension is extremely rare in these populations, and accelerates most rapidly around 40 years of age when hypertension is still uncommon. Into old age, as the prevalence of hypertension becomes widespread, the progression of fibroplasia abates. These details argue against hypertension as a cause of fibroplasia. 
Fig. 5. Each dot represents the mean of a population-specific 10year age group. Reproduced from [16].

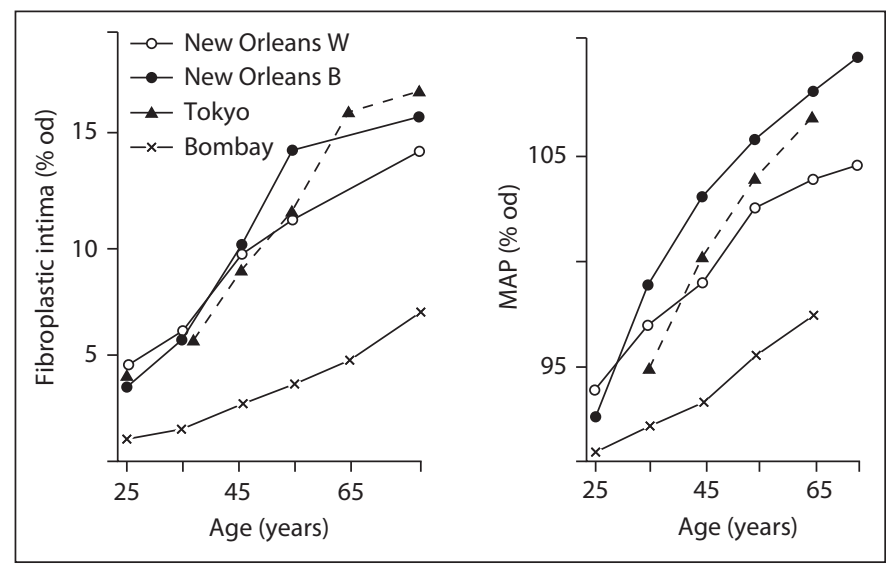

Figure 3 offers additional support for the view that hypertension does not accelerate fibroplasia because some subjects with many years of sustained hypertension can still be found at autopsy to have mild or minimal fibroplasia. It should be considered that the vascular features of malignant hypertension are reversible with therapeutic correction of the high blood pressure, but that the forerunning fibroplasia later revealed at autopsy is not [9]. This observation indicates that the effect of hypertension is to accelerate the malignant form of renal vasculopathy, but not the benign form. Malignant hypertension is a vicious cycle with hypertension accelerating vasculopathy, which then accelerates the hypertension. This closed cycle, if left untreated as in the past, goes rapidly to its fatal completion. If the benign condition were also a vicious cycle, then all hypertension would be malignant. It seems easier to accept that the cycle is not closed in the benign condition because hypertension does not accelerate the fibroplasia. The correlations seen in figures 2 and 5 are therefore most readily explained by nephron ischemia scattered heterogeneously through the renal cortex and raising blood pressure by Goldblatt mechanisms [21].

The Intersalt Study was a large collaborative study carried out in 52 populations around the world [25]. Its major conclusion was that urinary sodium excretion, a surrogate for dietary salt intake, was correlated between populations with the rise with age of systolic and diastolic pressures, but that the actual attained levels had no such correlation (omitting four exceptional primitive groups in Brazil, New Guinea and Kenya). The authors did not ask how the age-related rise correlated with the initial value, but their tabulations provide the data needed to assess the correlation [26]. The association showed no significant correlation, but rather revealed that the initial average systolic pressure in a population had no perceptible bearing on the average level attained at 55 years of age. This outcome offers evidence that higher pressure levels early in life do not affect the progression toward hypertension later in life.

It should also be considered that resistance level arteries in the human foot are among those most resistant to intimal fibroplasia, even though they are exposed to systolic pressures in the range of $300 \mathrm{~mm} \mathrm{Hg}$ for many hours each day while standing. Why hypertension should only have a harmful effect on arteries in a few organs, and especially the kidney, has rarely been discussed by advocates of the proposal that hypertension causes these arterial changes.

A logical consequence of the foregoing analysis is that blood pressure itself may not be a controlling influence upon the progression of intimal fibroplasia in renal interlobular arteries or upon its associated rise of MAP with age. Hence, if 
a risk factor is correlated with MAP, then it is unlikely to be a cause of fibroplasia. Salt intake, for instance, correlates with systolic and diastolic pressure, and the effect of salt increases progressively with rising levels of MAP [26]. That increment added by salt to the elevated MAP, like all contributors to MAP, can be viewed within the foregoing analysis as failing to accelerate the disease progression. Greater intakes of salt produce increments of elevated MAP at all ages from 20-60 years [27]. Hence, subjects with lifelong excesses in salt consumption should be exposed to several decades of elevated pressures. Yet, when at 50 years of age the salt is removed from their diets, the MAP quickly falls back to the levels of subjects never exposed to the salt effect [27]. Those many years of salt-induced MAP elevations leave no record within the relevant tissues of the body because removal of the salt obliterates its effect. The Intersalt finding that urinary sodium correlates across populations with the rise in systolic pressure on age can easily be explained by the exaggerated effect of salt at ever increasing levels of MAP. That is to say, populations that experience rising pressures are the populations with the most subjects who are salt-sensitive at 55 years of age. Presumably, removal of the salt from the diets of these subjects should obliterate the correlation found by Intersalt [27].

A similar logic applies to obesity. When obese subjects succeed in dietary weight loss, their blood pressures decline by the amount attributable to the weight change [26]. Again, many decades of pressure elevations induced by excessive body weight leave no record in the relevant body tissues because weight loss through dieting (and perhaps by bariatric surgery, which needs to be examined for this effect) fully returns pressures to levels seen in subjects who were never obese. Any other factor that acts to raise blood pressure can be seen in the same way. MAP itself is not apt to be a cause for fibroplasia (other than the malignant form) or the rise of MAP with age, therefore the increment of MAP induced by the factor in question is among those not having an effect on microscopic arteriosclerosis. The search for the etiology of benign essential hypertension should put less emphasis on the factors known to elevate MAP, and look elsewhere instead [28].

Analyses like those in figures 2 and 5 were also carried out for arteriolar hyalinization. The hyalinization was found to increase with age up to about age 40 years and then to level off at a constant range into old age [29]. Hence, the process is accelerated at ages when hypertension is rare (20-39 years), and fails to progress further at ages when hypertension is commonplace (ages 40-74 years). Also, the correlation across populations was not significant when relating hyalinization to MAP [16]. The correlation of arteriolar hyalinization with MAP across individuals is generally weak (in the range of $r=0.4$ ), and could be explained by an indirect correlation of hyaline in some way associated with accelerating arterial fibroplasia [12]. In a multivariate setting, fibroplasia correlates with MAP and hyalin loses its correlation with MAP, carrying nothing of additional significance. These findings favor the view that high MAP is not the driving force for arteriolar hyalinization, and that hyalinization does not induce sizable increases in blood pressure.

The curves shown in figure 5 can be described as fitting the equation $R_{C}=\beta A^{2}(1-0.1 A)$ [28]. This equation was accepted without statistically significant rejection in men and women of every population so far examined. Hence, the diversity in fibroplasia rates is governed by a single variable parameter, $\beta$. The values of $\beta$ so far observed around the world range from 0.0064 for men in Mexico to 0.0119 for women in Greenland. A similar equation for arteriolar hyalinization is $\mathrm{Hy}=\gamma \mathrm{A}^{2}(1-.0117 \mathrm{~A})$. The global range is from $\gamma=0.00098$ for women in Peru to $\gamma=0.00485$ for men in Greenland. The etiologic agents that account for these global variations are wholly unknown. Recognized agents such as salt, obesity and others are likely to contribute nothing of significance to this process (see above). These global variations could therefore be rich sources for clues to the mysterious etiologic agents, especially in migrant populations [28]. The finding that $R_{C}$ correlates with a related kind of intimal 
fibroplasia in coronary arteries might be of some help for this purpose [30].

In many various kinds of experimental models for hypertension, beginning historically with renal artery strictures, irritant wrapping of the kidneys or aldosterone-salt models and extended to later models $[8,11]$, the interlobular arteries readily develop the features of malignant hypertension. These include the hyperplastic mucinous edema of arteries (fig. 1d) and fibrinoid necrosis of arterioles (not illustrated here) along with characteristic changes in glomeruli, tubules and interstitium. Whether the characteristic fibroplasia of the benign human condition can also develop in these models is not clear. Most authors omit this feature from their descriptions of renal histology. Rare claims of finding appearances like those in figure $1 \mathrm{~b}$ raise the possibility that lesions of malignant hypertension might resolve and leave behind some alterations that resemble the human condition without actually replicating it in its natural form.

Textbooks of veterinary pathology put little emphasis on arteriosclerosis of any sort. Jubb and Kennedy [31], for instance, offer one short paragraph on sclerosis of visible arteries, and no mention of microscopic arteries or arterioles (other than of rare instances of hyalinization). The full range of domesticated animals apparently fail to manifest any significant degree of arteriosclerosis or arteriolosclerosis, and these processes are outside the range of interest of veterinarians. In a study of renal interlobular arteries in various mammalian species [32], it was found that the 3 -year-old rat had arteries that resemble those of 3-year-old humans, 6-year-old ferrets resemble 6-year-old humans, 12-year-old cats and dogs resemble 12-year-old humans, and 20-year-old horses and rhesus monkeys resemble 20-year-old humans. None of those species displayed anything resembling type 2 hypertension. If that type of hypertension can occur in animals, then it has to be experimentally induced, and the possibility of doing this has not been clearly demonstrated. The relationship of fibroplasia progression to age appears to be governed by the calendar and not by the longevity of each species.

Arteriolar hyalinization of afferent arterioles is readily induced in rats by administering antagonists of nitric oxide synthase such as L-NAME [33]. The effect of blood pressure on hyalinization, as distinct from glomerulosclerosis, however, has not been clarified.

\section{Conclusions}

The weight of evidence favors the view that type 1 hypertension does not evolve over time into type 2, indicating that elevated MAP does not accelerate the intimal fibroplasia of interlobular arteries in those individuals. Similarly, the intimal fibroplasia of interlobular arteries that is diagnostic of type 2 hypertension is most easily seen as a cause and not a consequence of elevated MAP. Agents such as salt and obesity can elevate MAP as long as the agents are actively in place, but their effects are readily reversible upon withdrawal of the agents. Hence, these agents do not appear to cause the rise of MAP with age, which introduces the high prevalence of type 2 hypertension in some aging populations. The search for etiology of benign essential hypertension should emphasize agents that are not on the usual lists of suspects.

\section{References}

1 Short D: The vascular fault in chronic hypertension. Lancet 1966;1:13021304.
2 Mulvany MJ, Baumbach GL, Aalkjaer C, Heagerty AM, Korsgaard $\mathrm{N}$, Schiffrin EL, Heistad DD: Vascular remodeling. Hypertension 1996;28:505-506.
3 Tracy RE, Overll EO: Arterioles of perfusion-fixed hypertensive and aged kidneys. Arch Path 1966:82:526-534. 
4 Folkow B, Gothberg G, Lundin S, Richsten SE: Structural 'resetting' of the renal vascular bed in spontaneously hypertensive rats (SHR). Acta Physiol Scand 1977;100:270-272.

5 Fishberg AM: The arteriolar lesions of glomerulonephritis. Arch Int Med 1927;40:80-97.

6 Geiger H, Fierlbeck W, Mai M, Ruchti H, Schönlfeld, Dämmrich J, Hugo C, Neumayer HH: Effects of early and late antihypertensive treatment on extracellular matrix proteins and mononuclear cells in uninephrectomized SHR. Kidney Int 1997;51:750-761.

7 Fishberg AM: Anatomic findings in essential hypertension. Arch Int Med 1925;35:659-668.

8 Stoddard LD, Puchtler H: Human renal vascular lesions and hypertension. Pathol Annu 1969;4:253-268.

9 McCormack LJ, Beland JE, Schneckloth RE, Corcoran AC: Effects of antihypertensive treatment on the evolution of the renal lesions in malignant nephrosclerosis. Am J Pathol 1958;34:1022-1018.

10 Jespersen B, Eiskjaer H, Christiansen NO, Olsen S, Pedersen EB: Malignant hypertension; relationship between blood pressure control and renal function during long-term observations of patients with malignant nephrosclerosis. J Clin Hypertens 1987;3:409-418.

11 Helmchen U, Bohle RM, Kneissler U, Groene HJ: Intrarenal arteries in rats with early two-kidney, one clip hypertension. Hypertension 1984;6:III87III92.

12 Tracy RE, Parra D, Eisaguirre W, Balanza AT: Influence of arteriolar hyalinization on arterial intimal fibroplasia in the renal cortex of subjects in the United States, Peru, and Bolivia, applicable also to other populations. Am J Hypertens 2002;15:1064-1073.

13 Tracy RE: Age trends of renal arteriolar hyalinization explored with the aid of serial sections. Nephron Clin Pract 2007;105: c171-c177.
14 Ljungqvist A: The intrarenal arterial pattern in essential hypertension; a micro-angiographic and histological study. J Path Bact 1962;84:313-325.

15 Tracy RE, Ishii T: What is 'nephrosclerosis'? Lessons from Japan and Mexico. Nephrol Dial Transplant 2000;15:1357-1366.

16 Tracy RE: The heterogeneity of vascular findings in the kidneys of patients with benign essential hypertension. Nephrol Dial Transplant 1999;14:1634-1639.

17 Sommers SC, Relman AS, Smithwick RH: Histologic studies of kidney biopsy specimens from patients with hypertension. Am J Pathol 1958;34:685-702.

18 Pontremoli RP, Viazzi F, Martinoli C, Ravera M, Nicolella C, Berruti V, Leoncini G, Ruello N, Zagami P, Bezante GP, Derchi LE, Deferrari $\mathrm{G}$ : Increased renal resistive index in patients with essential hypertension: a marker of target organ damage. Nephrol Dial Transplant 1999;14:360-365

19 Hollenberg NK, Epstein M, Basch RI, Merrill JP: 'No man's land' of renal vasculature; an arteriographic and hemodynamic assessment of the interlobar and arcuate arteries in essential and accelerated hypertension. Am J Med 1969;47:845-854.

20 Ladefoged J: Measurements of the renal flood flow in man with the ${ }^{133}$ xenon wash-out technique. Scand J clin Lab Invest 1966;18:299-315.

21 Sealy JE, Blumenfeld JD, Bell GM, Pecker MS, Sommers SC, Laragh $\mathrm{JH}$ : On the renal basis for essential hypertension: nephron heterogeneity with discordant renin secretion and sodium excretion causing a hypertensive vasoconstriction-volume relationship. J Hypertens 1988;6:763777

22 Gatta A, Merkel C, Pessina AC, Milani L, Sacerdoti D, Zuin R: Renal hemodynamics in essential hypertension assessed by 133 -xenon washout and selective renal angiography. Angiology 1982;33:818-824.
23 Aukland K: Intrarenal distribution of blood flow. Are reliable methods available for measurements in man? Scan J Clin Lab Invest 1975;35:481486.

24 Tracy RE, Lanjewar DN, Ghorpade KG, Valand AG, Raghuwanshi SR: Renovasculopathies of hypertension in Bombay, India. Geriat Nephrol Urol 1997;7:101-109.

25 Intersalt Cooperative Research Group: Intersalt: an international study of electrolyte excretion and blood pressure. Results for 24 hour urinary sodium and potassium excretion. BMJ 1988;297:319-328.

26 Tracy RE: Salt, obesity, and alcohol fail to induce a lasting rise of blood pressure with age, and may be independent of renocortical vasculopathy. QJM 1999;92:601-607.

27 Law MR, Frost CD, Wald NJ: By how much does salt reduction lower blood pressure? III - analysis of data from trials of salt reduction. BMJ 1991;302:819-823.

28 Tracy RE, Guileyardo JM: Renovasculopathies of hypertension in Hispanics of Dallas, Texas. Arch Med Res 1999;30:40-48.

29 Tracy RE, Parra D, Eisaguirre W, Balanza AT: Influence of arteriolar hyalinization on arterial intimal fibroplasia in the renal cortex of subjects in the United States, Peru, and Bolivia, applicable also to other populations. Am J Hypertens 2002;15:1064-1073.

30 Tracy RE: Introducing the sequestration hypothesis; in Tracy RE (ed): The Role of Aging in Atherosclerosis. Dordrecht, Kluwer Academic Publishers, 2003, pp 1-10.

31 Jubb KVF, Kennedy PC: Pathology of domestic animals. New York, Academic Press, 1970.

32 Tracy RE, Johnson LK: Aging of a class of arteries in various mammalian species in relation to life span. Gerontology 1994;40:291-297.

33 Ono H, Saitoh M, Ono Y, Ishimitu T, Matsuoka H: Imidapril improves L-NAME-exacerbated nephrosclerosis with TGF- $\beta 1$ inhibition in spontaneously hypertensive rats. J Hypertens 2004;22:1389-1395.

Richard E. Tracy, MD, PhD, Professor Emeritus

Department of Pathology, Louisiana State University Health Sciences Center

1901 Perdido St, 1 P5

New Orleans, LA 70112 (USA)

Tel. +1 504568 6072, Fax +1 504568 6037, E-Mail rtracy@lsuhsc.edu 